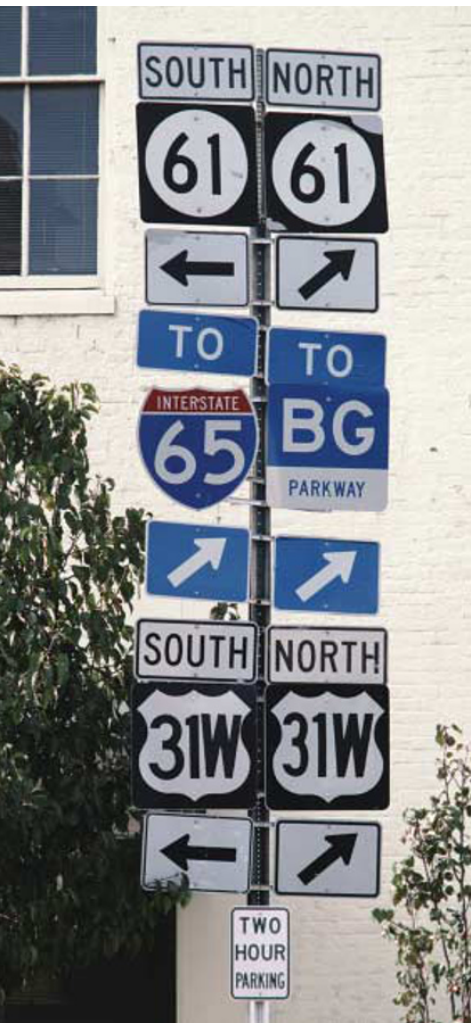

\title{
Signalling complexities for Pseudomonas
}

A new paper in Proceedings of the National Academy of Sciences USA has uncovered a previously unknown activity of the phytopathogen Pseudomonas syringae: the ability to induce systemic-induced susceptibility (SIS) to subsequent infection, and has also identified the small molecule required to do so.

Generally, plants respond to an attack by microbial pathogens by inducing salicylic acid (SA)-dependent systemic resistance pathways — systemic acquired resistance (SAR) — whereas the resistance pathways induced in response to an attack by insect herbivores are jasmonic acid (JA)-dependent. It is known that the crosstalk between the two pathways, which can be additive or antagonistic, is complex and varies depending on the pathogens and plant hosts involved.

In previous work, a model system involving Arabidopsis, P. syringae and an insect herbivore, the cabbage looper (Trichoplusia ni), was established to investigate how infection by a bacterial pathogen affects the response of the plant to subsequent attack by other bacterial pathogens and insect herbivores. The results obtained suggested the presence of two signalling pathways, one of which was correlated with SAR and enhanced resistance to $T$. ni feeding and a second that enhanced susceptibility to T. ni feeding via an unknown signal.

In this latest work, infection of Arabidopsis lower leaves with virulent strains of $P$. syringae showed a small but reproducible SIS effect, with enhanced secondary growth of $P$. syringae in uninfected upper leaves. Further analysis pointed to the phytotoxin coronatine (COR), an important $P$. syringae virulence factor and a mimic of JA, as the molecule responsible for this effect. The authors hypothesized that the effects of COR could be mediated by antagonism of the SA-dependent SAR response. Experimental work showed that avirulent nonCOR-producing strains elicited a stronger SAR response than did avirulent COR-producing strains, suggesting that COR could function by interfering with SA-dependent signalling.

The authors also analysed the effects of COR on insect herbivory, and interpret their observations as

\section{Competitive signalling}

$\mathrm{N}$-acylhomoserine lactones, better known as AHLs, are the quorum-sensing molecules that Gram-negative bacteria use to coordinate cell-density-dependent processes, which include virulence, biofilm formation and antibiotic synthesis. A new study published in Proceedings of the National Academy of Sciences USA shows that these versatile molecules can also function as antimicrobials.

Studying the coordinated light production of the glowing bacterial symbionts that populate the light organ of the squid led to a realization that Gram-negative bacteria use diffusible signalling molecules to coordinate population behaviour and behave as multicellular groups. These signalling molecules, named quorum-sensing hormones or quormones, are produced by many Gram-negative bacteria. Intense research in the past 10 years has led to the elucidation of quormone biosynthetic pathways and an understanding of the regulatory functions of quormones.
AHLs, one class of quormone, are produced by several important Gramnegative animal and plant pathogens, including Pseudomonas aeruginosa, which can infect cystic fibrosis sufferers to cause debilitating opportunistic infections. While investigating how long AHLs can persist in an aqueous environment, Kaufmann et al. discovered that one of the AHLs produced by $P$. aeruginosa, $N$-(3-oxododecanoyl) homoserine lactone, was non-enzymatically and spontaneously converted into a tetramic acid. Importantly, this wasn't a peculiarity of $\mathrm{N}$-(3-oxododecanoyl) homoserine lactone, and tetramic acids were produced by a selection of variant chainlength AHLs that Kaufmann et al. tested.

Using bioassays, the tetramic acid produced was shown to be cytotoxic - at concentrations that would be present in biofilms formed by $P$. aeruginosa - but only towards Gram-positive bacteria, including Bacillus, Streptococcus and Listeria species. Surprisingly, $\mathrm{N}$-(3-oxododecanoyl) homoserine lactone was also mildly cytotoxic towards some of the Gram-positive strains tested. Both the AHL and the tetramic acid are much less potent than other antimicrobials in clinical use, but, nonetheless, their activity might give the producer organism a competitive advantage in a mixed community.

Tetramic acids also bind metals, although the effects of metal chelation on their cellkilling functions are variable. The AHLderived tetramic acid identified in this study chelated $\mathrm{Fe}^{3+}$, and although the tetramic acid didn't bind the metal as tightly as pyoverdin, the main siderophore of P. aeruginosa, it had higher affinity for iron than pyochelin, a second siderophore found in this species, so tetramic acid might function both as an iron scavenger and as an antimicrobial.

These interesting observations need to be related to the behaviour of AHL-producing bacteria in mixed communities, but it seems that when a quorum is reached, the signals that are produced might regulate multiple phenotypes and allow Gram-negative producer species to gain a competitive advantage in communities.

Susan Jones

(2) References and links ORIGINAL RESEARCH PAPER Kaufmann, G. F. et al. Revisiting quorum sensing: discovery of additional chemical and biological functions for 3-oxo- $N$-acylhomoserine lactones. Proc. Natl Acad. Sci USA 102, 309-314 (2005) WEB SITE

Kim Janda's laboratory:

http://www.scripps.edu/chem/janda/ 
indicating that virulent $P$. syringae also induces systemic susceptibility to $T$. ni herbivory but not via COR. In fact, it was found that COR induces systemic resistance to T. $n i$, consistent with its role as a JA mimic.

So, this work has revealed a role for COR in $P$. syringae-mediated manipulation of plant systemic defences and has also confirmed that the interactions involved in these defences are extremely complex. Future work will provide further details on the role of COR and its interactions with the SA- and JAmediated pathways, as well as continuing to analyse the molecular mechanisms responsible for P. syringae-mediated susceptibility to insect herbivores.

Sheilagh Molloy

\section{(2) References and links} ORIGINAL RESEARCH PAPER Cui, J. et al. Pseudomonas syringae manipulates systemic plant defences against pathogens and herbivores. Proc. Natl Acad. Sci. USA 18 Jan 2005 (doi:10.1073/pnas.0409450102)

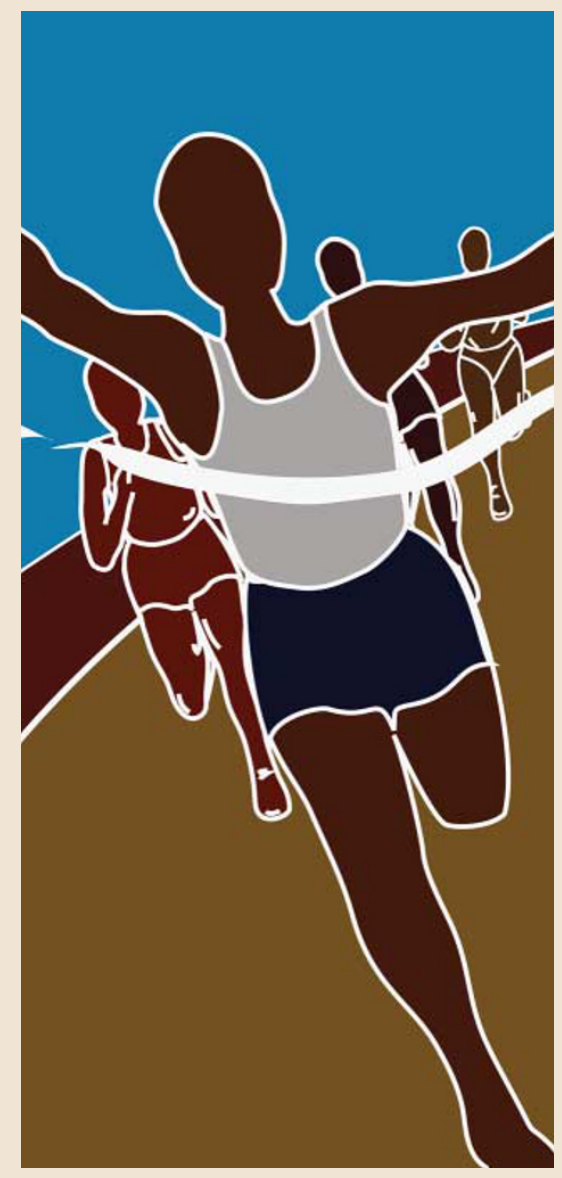

BACTERIAL PHYSIOLOGY

\section{A function for redundancy}

The availability of genome sequences promises to enable researchers to understand the physiology of individual cells, but when there are two or more pathways for metabolism of a substrate - known as metabolic modules - it takes more than 'omics to probe which pathway is used and when. Marx et al. used flux analysis to show that methylobacteria metabolize formaldehyde by routing it through two different pathways, and report their findings in the latest issue of PLoS Biology.

Methylobacteria are facultative methylotrophs, meaning that these bacteria can grow on carbon compounds that have one or more carbon atoms. Methylotrophs can grow on methanol, which is important because plants produce methanol, and plant-bacterial associations might affect seed germination and plant development.

Growing on methanol presents methylotrophs with a problem - the central intermediate in methanol catabolism is formaldehyde, which is toxic. In methylotrophs, formaldehyde can be converted into serine, and shuttled into central metabolism by one of two routes that are found only in these bacteria - a direct route (green) or a long route (blue) (see figure). The direct route is a non-enzymatic reaction that combines formaldehyde with tetrahydrofolate to generate methylene- $\mathrm{H}_{4} \mathrm{~F}$, whereas the long route consumes one molecule of ATP and involves several enzymecatalysed steps. A third module found in many bacteria oxidizes formaldehyde to $\mathrm{CO}_{2}$. Genetics indicate that both the direct and the long routes are required for growth on formaldehyde, but why does Methylobacterium extorquens have redundant metabolic modules? Marx et al. have tested this directly by monitoring the metabolism of methanol using stable-isotope and radioisotopelabelling approaches.

By growing M. extorquens on methanol labelled with deuterium $\left(\mathrm{CD}_{3} \mathrm{OD}\right)$ they were able to unravel which module was used in different growth conditions, since the direct route incorporates 1 deuterium atom in each serine molecule, while the long route incorporates 2 deuterium atoms in each serine. By measuring the ratios of labelled serine produced they found that metabolism of formaldehyde by the long route dominated when succinate-grown cells were first fed methanol, but that after an acclimation period the direct route was favoured. Although both routes operate, they are used differentially dependent on the growth conditions.

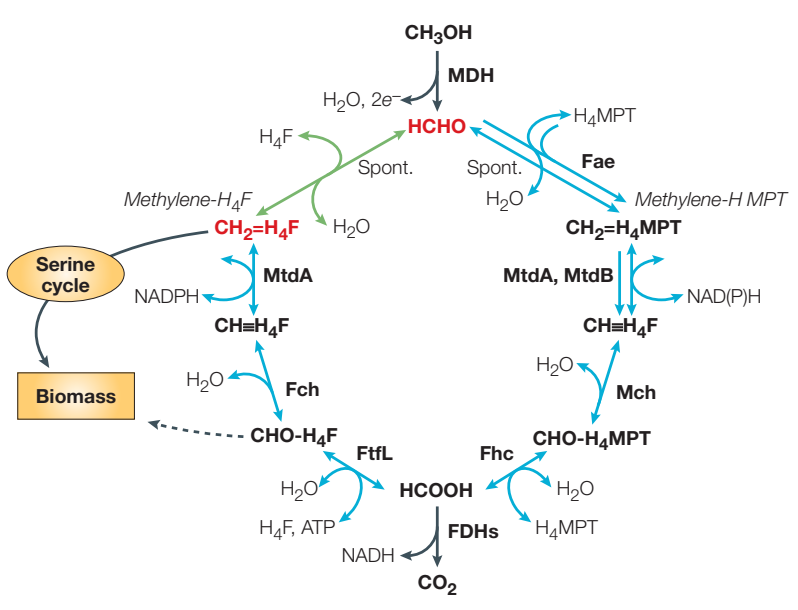

Image modified from Marx et al. PLoS Biol. (2005)

Flux of carbon through metabolism was also monitored using ${ }^{14} \mathrm{C}$-labelled $\mathrm{CH}_{3} \mathrm{OH}$ and by analysing the rates of methanol oxidation, assimilation of $\mathrm{Cl}$ units and $\mathrm{CO}_{2}$ production. This analysis confirmed that the long route is initially used when cells are transferred to methanol, and that although the contribution of this route quickly declines, it is still considerable. A mathematical model produced using known kinetic parameters for the enzymes in formaldehyde metabolism was also devised. By simulating a switch from growth on succinate to methanol, the model predicted the same switch from the long to the direct route that was found using flux analysis. Marx et al. endeavoured to delete the $\mathrm{ft} f \mathrm{~L}$ gene, an intrinsic part of the long route, reasoning that if the long route is only necessary for acclimation to methanol growth, it might be possible to find mutants if the bacteria were already growing on methanol. The failure of this strategy indicates that both routes are needed, even when cells are continuously grown on methanol.

This research shows that biochemistry must go hand-in-hand with genomics to create useful models to understand cell physiology. Switching between different metabolic modes and metabolizing toxic intermediates isn't restricted to bacteria, so this research could represent a new paradigm for growth in toxic environments.

Susan Jones

\section{(1) References and links}

ORIGINAL RESEARCH PAPER Marx, A. et al. Flux analysis uncovers key role of functional redundancy in formaldehyde metabolism. PLoS Biol. 3, e16 (2005)

WEB SITE

Mary Lidstrom's laboratory:

http://www.hhmi.org/research/professors/lidstrom_bio.html 